Military Technical College

Kobry El-Kobbah, Cairo, Egypt

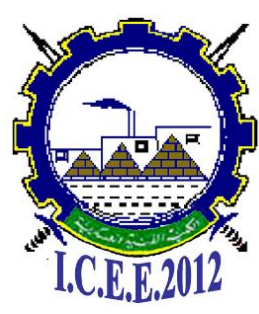

$6^{\text {th }}$ International Conference on

Chemical \& Environmental Engineering 29 -31 May, 2012.

\title{
PREPARATION AND CHARACTERIZATION OF NOVEL BINDER FOR COMPOSITE SOLID ROCKET PROPELLANT
}

\author{
Hosam E. Mostafa*, I. M. Kamal ${ }^{*}$
}

\begin{abstract}
Azido hydroxy terminated polybutadiene (AHTPB) is a candidate energetic binder for composite propellants having the same properties of hydroxy terminated polybutadiene (HTPB) but has an advantage over HTPB concerning its energetic property due to presence of azide group. Thermochemical calculations were done with different AHTPB percentages and the reference HTPB to examine the effect of binder change on the specific impulse and the flame temperature which are from the important parameters for the propellant performance and gun barrel age. AHTPB prepolymer was prepared by two steps method in which hydroxy terminated polybutadiene (HTPB) is firstly saturated with bromine $\left(\mathrm{Br}_{2}\right)$ at room temperature giving bromo hydroxy terminated polybutadiene (BHTPB). Secondly, the formed BHTPB undergo substitution reaction using sodium azide $\left(\mathrm{NaN}_{3}\right)$ at $70^{\circ} \mathrm{C}$ for 30 hours on batches giving AHTPB with density of $0.967 \mathrm{~g} / \mathrm{cm}^{3}$ and viscosity of about $5000 \mathrm{cP}$. The prepolymer was successfully prepared with conversion of (15\%) and characterized by using some techniques such as infrared spectroscopy, elemental analysis, water content measurements and milligram equivalent $(\mathrm{OH})$.
\end{abstract}

\section{Key Words}

Azido hydroxy terminated polybutadiene, Binder Systems, thermochemical calculations, preparation of AHTPB, Caracterization of Prepolymer, Composite solid rocket propellants.

\footnotetext{
* Egyptian Armed Force
} 


\section{Introduction}

Design of future weapon systems requires using modern types of explosives and propellant formulations having enhanced performance and reduced vulnerability during storage and transportation. Several important design considerations for such formulations include improved mechanical properties, decreased plum signature, extended service life and reduced environment impact during manufacture, utilization and disposal [1].

Composite propellants are made of a polymeric matrix, loaded with a solid powder oxidizer, and possibly a metal powder that plays the role of a secondary fuel component. The first composite propellants used thermoplastic binders such as asphalt, polyvinyl chloride, and polyisobutylene which require softening or melting a temperature increase. Around 1950, the first liquid binders allowing crosslinking appeared. From 1950 until 1965, composite propellants were made with polysulifide (thiokols) and polyurethane polymers. From 1965 on, new binders emerged, with a functional polybutadiene basis: acrylonitrile-acrylic acidbutadiene, acrylic acid butadiene copolymer, and homopolymers with functional ends called telechelics. These polymers led to increasingly better performing elastomeric binders, since they offered higher solids loadings and a wider operating temperature range, especially at low temperatures and good processing but, they are inert binders having low ballistic performance. So, the use of new energetic binders has emerged. Azido hydroxy terminated polybutadiene (AHTPB) is useful as an energetic prepolymer in plastic bonded explosives and in rocket propellants to enhance the propellant performance, stability and keeping high solids loading, wider operating temperature range especially at low temperatures and good processing [2].

AHTPB prepolymer was prepared by two steps method in which hydroxy terminated polybutadiene (HTPB) dissolved in normal hexane is firstly saturated with bromine $\left(\mathrm{Br}_{2}\right)$ also dissolved in normal hexane at room temperature giving bromo hydroxy terminated polybutadiene (BHTPB).Secondly, the formed BHTPB undergo substitution reaction using sodium azide $\left(\mathrm{NaN}_{3}\right)$ at which the $\left(-\mathrm{N}_{3}\right)$ group substitute (-Br) group at $70{ }^{\circ} \mathrm{C}$ for 30 hours on batches. The introduction of the azide group to the HTPB increase its energy and so increases the performance of the propellant formed by the new AHTPB keeping the physical and chemical properties of HTPB which is suitable to be used in composite propellant. A full series of characterizations was being done to examine the product through physical, chemical and instrumental analysis. Thermochemical calculations were performed on some formulations to measure the effect of aizde group introduction on the propellant characteristics $[3,4]$.

\section{Thermochemical Calculations}

Thermochemical calculations have been used with considerable success as a fast and effective tool to investigate the characteristics of different types of propellant formulations. Thermochemical calculation is an essential step for choosing propellant formulations that are candidate for a given practical application and to minimize many costly experiments which are used to evaluate the performance and properties of new propellant formulations $[5,6]$.

The effect of the content of prepared AHTPB and the reference binder HTPB on the specific impulse and adiabatic flame temperature of the composite solid rocket propellant formulations containing the prepared prepolymer was examined by using a special computer program. Some formulations were selected with changing the binder content from $10 \%$ weight percent to $20 \%$ to determine the effect of variation of binder content on the propellant characteristics. In these formulations, the dioctyl azalate DOZ was selected as the plasticizer for the prepolymers HTPB and AHTPB. Its content was taken as $25 \%$ based on the used weight of prepolymer. Hexamethelene Diisocyanates HMDI was selected as the curing agent 
in mg. equivalent $\mathrm{NCO} / \mathrm{g}$ of $\mathrm{HMDI}=11.9$ and the ratio of $\mathrm{NCO} / \mathrm{OH}$ was selected to be $=0.9$. The calculated summary formula (S.F) and the enthalpy of formation $\left(\Delta \mathrm{H}_{\mathrm{f}}\right)$ of one $\mathrm{kg}$ of polyurethane binder based on HTPB and AHTPB were listed in table (1).

The content of different ingredients in composite solid rocket propellant formulations containing polyurethane binder based on HTPB prepolymer and AHTPB, and the calculated values of specific impulse $I_{s}$ and adiabatic flame temperature $T_{f}$ of these formulations are listed in table (2,3). The dependence of the calculated values of $\mathrm{I}_{\mathrm{sp}}$ for all formulations $\mathrm{F} 1$ F22 on the binder content is illustrated in figure (1) while the effect of the binder content on the calculated values of $T_{f}$ for the same formulations are shown in figure (2). It was found that the $I_{s p}$ and $T_{f}$ decreases by the increase of the binder content. By discussing $I_{s p}$ and $T_{f}$ at the optimum binder percentage which was (14\%), it is found that the $\mathrm{I}_{\mathrm{sp}}$ was increased from 240s for HTPB to $253 \mathrm{~s}$ for AHTPB and so the flame temperature was increased from $3405 \mathrm{~K}$ to $3490 \mathrm{~K}$ which the gun barrel can withstand. Results of thermochemical calculations were very important for propellant compositions that are candidate for preparation and expected to give the required performance of the needed application.

Table (1) Summary formula and $\left(\Delta \mathbf{H}_{\mathrm{f}}\right)$ of one $\mathrm{kg}$ PU binder based on HTPB or AHTPB and that of basic ingredients

\begin{tabular}{|l|c|c|c|c|c|}
\hline \multirow{2}{*}{$\begin{array}{l}\text { Binder } \\
\text { Ingredients }\end{array}$} & \multirow{2}{*}{$\begin{array}{c}\mathrm{H} \\
\mathbf{f} \mathbf{f}\end{array}$} & \multicolumn{4}{|c|}{ Summary formula / kg } \\
\cline { 3 - 6 } & $\mathbf{k c a l} / \mathbf{k g}$ & $\mathbf{C}$ & $\mathbf{H}$ & $\mathbf{O}$ & $\mathbf{N}$ \\
\hline HTPB & 65.00 & 74.07 & 111.11 & - & - \\
\hline AHTPB & 62.500 & 73.26 & 109.89 & - & 10.99 \\
\hline DOZ & -880.30 & 60.58 & 116.32 & 9.693 & - \\
\hline HMDI & -351.60 & 46.92 & 70.381 & 11.73 & 11.73 \\
\hline PU Based on HTPB & -135.43 & 70.20 & 110.54 & 2.425 & 0.582 \\
\hline PU Based on AHTPB & -136.00 & 69.60 & 109.25 & 2.382 & 8.925 \\
\hline
\end{tabular}

Table (2) Specific impulse and flame temperature of HTPB-CSRP formulations

\begin{tabular}{|l|c|c|c|c|c|c|c|c|c|c|c|}
\hline $\begin{array}{c}\text { Formulation } \\
(\%)\end{array}$ & $F_{1}$ & $F_{2}$ & $F_{3}$ & $F_{4}$ & $\mathbf{F}_{5}$ & $F_{6}$ & $F_{7}$ & $F_{8}$ & $F_{9}$ & $F_{10}$ & $F_{11}$ \\
\hline HTPB & 10.00 & 11.00 & 12.00 & 13.00 & $\mathbf{1 4 . 0 0}$ & 15.00 & 16.00 & 17.00 & 18.00 & 19.00 & 20.00 \\
\hline APC & 73.06 & 72.25 & 71.44 & 70.62 & $\mathbf{6 9 . 8 0}$ & 69.00 & 68.19 & 67.38 & 66.56 & 65.75 & 64.90 \\
\hline Al powder & 16.94 & 16.75 & 16.56 & 16.38 & $\mathbf{1 6 . 2 0}$ & 16.00 & 15.81 & 15.62 & 15.44 & 15.25 & 15.10 \\
\hline $\mathrm{I}_{\mathrm{S}}(\mathrm{s})$ & 269.3 & 267.3 & 265.6 & 254.6 & $\mathbf{2 4 0 . 4}$ & 234.3 & 228.1 & 221.8 & 216.6 & 213.3 & 212.0 \\
\hline $\mathrm{T}_{\mathrm{f}}(\mathrm{K})$ & 3677 & 3631 & 3571 & 3494 & $\mathbf{3 4 0 4}$ & 3344 & 3275 & 3178 & 3070 & 2971 & 2900 \\
\hline
\end{tabular}

Table (3) Specific impulse and flame temperature of AHTPB-CSRP formulations

\begin{tabular}{|l|l|l|l|l|l|l|l|l|l|l|l|}
\hline $\begin{array}{l}\text { Formulation } \\
(\%)\end{array}$ & $F_{12}$ & $F_{13}$ & $F_{14}$ & $F_{15}$ & $\mathbf{F}_{16}$ & $F_{17}$ & $F_{18}$ & $F_{19}$ & $F_{20}$ & $F_{21}$ & $F_{22}$ \\
\hline AHTPB & 10.00 & 11.00 & 12.00 & 13.00 & $\mathbf{1 4 . 0 0}$ & 15.00 & 16.00 & 17.00 & 18.00 & 19.00 & 20.00 \\
\hline APC & 73.06 & 72.25 & 71.44 & 70.62 & $\mathbf{6 9 . 8 0}$ & 69.00 & 68.19 & 67.38 & 66.56 & 65.75 & 64.90 \\
\hline Al powder & 16.94 & 16.75 & 16.56 & 16.38 & $\mathbf{1 6 . 2 0}$ & 16.00 & 15.81 & 15.62 & 15.44 & 15.25 & 15.10 \\
\hline $\mathrm{I}_{\mathrm{S}}(\mathrm{s})$ & 268.1 & 268.5 & 269.3 & 263.3 & $\mathbf{2 5 3 . 0}$ & 239.7 & 233.8 & 228.2 & 222.6 & 217.5 & 212.7 \\
\hline $\mathrm{T}_{\mathrm{f}}(\mathrm{K})$ & 37067 & 3664 & 3621 & 3562 & $\mathbf{3 4 9 0}$ & 3405 & 3349 & 3289 & 3200 & 3102 & 2995 \\
\hline
\end{tabular}




\section{Experimental}

\section{Preparation of AHTPB}

The apparatus used for preparation of AHTPB was designed to get the best control of the reaction and the highest possible conversion. It consists of water bath, 2 liter conical flask, magnetic stirrer, thermostat head, and reflux and nitrogen blanket. AHTPB was prepared on two steps reaction. Addition (bromination) step then substitution (azidation) step and they proceed as follows:

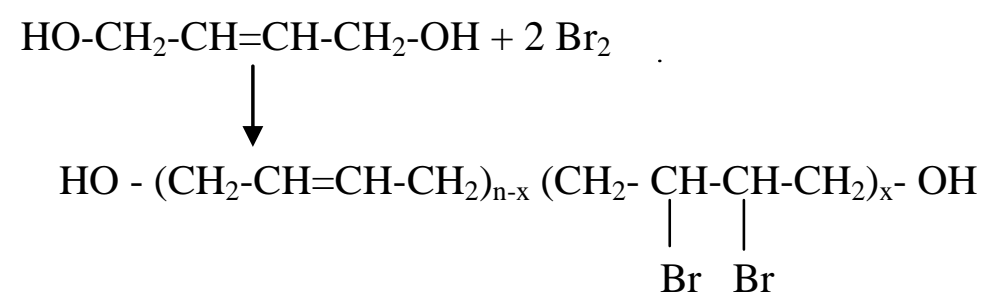

The first step was bromination at which HTPB dissolved in n-hexane at normal temperature under agitation until it was completely dissolved forming homogenous solution. $\mathrm{Br}_{2}$ together with $\mathrm{n}$-hexane were placed in a suitable conical flask, which was closed to minimize the hazards of bromine. The $\mathrm{Br}_{2}$ solution was transferred to a dropping funnel, then start dropping the solution drop by drop at normal temperature while stirring to prevent the agglomeration of the dissolved polymer. It was found that the red color of the bromine vanish as it reacted forming BHTPB.<smiles>NC(CCCC=CCCO)C(N)COO</smiles>

The second step was the substitution step at which the azide group $\left(-\mathrm{N}_{3}\right)$ substitute the (-Br) in the HTPB molecule. The BHTPB solution together with $\mathrm{NaN}_{3}$ was transferred to the reactor. The mixture was heated at $70^{\circ} \mathrm{C}$ under reflux for 30 hour on batches until the reaction was completed. While the mixture cooled down tell a white precipitate of sodium bromide appears, it was filtered using an ordinary filter paper. No washing took place because all the salts was removed as AHTPB was dissolved in n-hexane, then drying took place in a rotary evaporator to get rid of water and solvent by adjusting it at $60^{\circ} \mathrm{C}$ and pressure 20 mbar for 2-3 hours. Then, employ severe conditions by increase of temperature to $60^{\circ} \mathrm{C}$ for 15 minutes and the polymer is transferred to vacuum furnace at $40^{\circ} \mathrm{C}$ for 24 hours. A honey like shape amber color AHTPB was produced [7, 8]. 


\section{Characterization of the Prepared AHTPB}

The product was characterized through physical and chemical analysis by determining the $(\mathrm{OH})$ group as it is very important in the propellant curing, density which affect the volumetric loading of the propellant and so the performance, viscosity which play the main role in propellant processing, and also through instrumental analysis using FTIR principle to determine whether the azide group was introduced in the structure of HTPB, elemental analysis to determine the percentage of nitrogen content which express the energy of the propellant as nitrogen content increase the propellant become more energetic and water content is very important in the curing of propellant.

\section{Physical and chemical characterization}

\section{Determination of $(\mathrm{OH}) \mathrm{mg}$ Equivalent}

The determination of the mg equivalent is very important because curing of solid rocket propellant formulations is fully dependant on it together with NCO groups (curing ratio) as the two groups forms the urethane group which is the matrix that held all the ingredients of the propellant together [8].

Value of $(\mathrm{OH}) \mathrm{mg}$ equivalent of was determined according to following equation:

Where:

$$
\text { mg. eqv. } \mathrm{OH} / \mathrm{g} \text { binder }=\left[\left(\mathrm{V}_{2}-\mathrm{V}_{1}\right) * \mathrm{~N}\right] / \mathrm{W}
$$

$\mathrm{V}_{1} \ldots$. Volume in $\mathrm{cm}^{3}$ of $\mathrm{NaOH}$ solution required for titration of blank.

$\mathrm{V}_{2} \ldots .$. Volume in $\mathrm{cm}^{3}$ of $\mathrm{NaOH}$ solution required for titration of sample.

N..... Normality of $\mathrm{NaOH}$ solution.

W.....Weight of sample in $\mathrm{g}$.

\section{Determination of Density}

Density of the prepared AHTPB samples was determined by selecting an ordinary densimeter in the predicted range, inserting it in the measuring cylinder filled with sample and leaving it to reset and then take the value of density.

\section{Determination of Viscosity}

Brookfield viscometer was used for viscosity measurements of the prepared AHTPB samples. The process of selecting the spindle and the speed for an unknown fluid is normally trial and error. An appropriate selection will result in $(10-100 \mathrm{cP})$ on the instrument scale. Two general rules will help in the trial and error process $[9,10]$ :

Viscosity range is inversely proportional to the size of the spindle.

Viscosity range is inversely proportional to the rotational speed.

\section{Instrumental characterization}

\section{Infrared Spectroscopy}

Shimadzu 8000 Fourier transformation infrared (FTIR) was used to detect the presence of the azide group $\left(-\mathrm{N}_{3}\right)$ in the prepared AHTPB sample, which is an indication of the azidation reaction and the appearance of AHTPB. In case of viscous liquids as AHTPB samples, a thin layer of the polymer was placed on the surface of the $\mathrm{KBr}$ tablet. The used wave number range was $500-4000 \mathrm{~cm}^{-1}$, which is the appropriate range for most organic compounds. It is 
well known that IR spectra of the organic compounds can be divided into three main regions; functional group region $4000-1300 \mathrm{~cm}^{-1}$, finger print region $1300-910 \mathrm{~cm}^{-1}$ and aromatic region from $910-500 \mathrm{~cm}^{-1}[11]$.

\section{Elemental Analysis}

PERKIN-ELMER $240 \mathrm{CHN}$ elemental analyzer with AD-4 auto balance was used to detect the percentage of $\mathrm{C}, \mathrm{H}$ and $\mathrm{N}$ in the prepared samples which facilitates the detection of nitrogen content taken as a reference to the explosophorous azide group, responsible for the excess energy of the energetic prepolymer. Obtained results make it possible to compare the theoretical and determined experimental percentage of $\mathrm{C}, \mathrm{H}$ and $\mathrm{N}$ in the prepared samples [12].

\section{Determination of Water Content}

Water content is an important property especially if we need to use AHTPB as an ingredient in composite propellant formulations. Karl-Fisher technique was used for determination of the water content of the prepared samples. $200 \mathrm{~cm}^{3}$ of titrating solvent was added into the 500 $\mathrm{cm}^{3}$ beakers with reagent until the end-point using the diluted and standardized reagent. Then, about $2.5 \mathrm{~g}$ of the sample were added. Repeat the titration again with diluted standardized Karel- Fisher reagent, to the end point. The water content (\% by weight) was calculated as follows [13]:

K.F reagent..... Karel - Fisher $\left(\mathrm{cm}^{3}\right)$

$$
\text { Water content } \% \text { by weight }=\frac{K . F_{\text {reagent }} * \text { titer } * 100}{\text { wieght }_{\text {sample }}}
$$

Titer.... Ratio of the employed water $(\mathrm{g}) /$ volume of K.F reagent.

W...... weight (g)

\section{Results and Discussions}

\section{Preparation of AHTPB}

AHTPB was successfully prepared by two-steps method mentioned before. Three trials were done to examine the conversion, it was noticed that the conversion was increased by increasing the amount of sodium azid, but over a certain limit agglomerations and precipitations took place and the colour was changed and no product was formed. The best conversion was found to be $(15 \%)$.

\section{Physical and Chemical Characterization Hydroxyl number (OH)}

The $(\mathrm{OH}) \mathrm{mg}$. equivalent of HTPB was $(0.8541)$ while the $(\mathrm{OH}) \mathrm{mg}$. equivalent $\mathrm{OH} / \mathrm{g}$ AHTPB was found to be (0.8516) indicating that the chemical reactions carried on HTPB to produce AHTPB did not affect the content of terminal hydroxyl group [14].

\section{Density}

Density of AHTPB was found to be about $0.967 \mathrm{~g} / \mathrm{cm}^{3}$ and it was a promising value as it was higher than that of HTPB which was $0.903 \mathrm{~g} / \mathrm{cm}^{3}$, giving higher volumetric loading in the rocket propellant manufacture field. 


\section{Determination of Viscosity}

The viscosity of the sample was determined using Brookfield viscometer. Viscosity of AHTPB was found to be about $(5039 \mathrm{cP})$ which was slightly lower than that of HTPB (5306 $\mathrm{cP}$ ) but it was a promising value for rocket propellant processing.

\section{Instrumental characterization}

\section{Infrared Spectroscopy}

The azide as a functional group and more significantly as stretching vibration was inserted into the AHTPB structure as appears on figure (3) due to the existence of the absorption band at $2100.3 \mathrm{~cm}^{-1}$ which characterize the azide group. The halide peaks still appear at $499 \mathrm{~cm}^{-1}$ and $518 \mathrm{~cm}^{-1}$ as the halide group appear at the region from $600-500 \mathrm{~cm}^{-1}$ the presence of halide traces indicate that the substitution of azide group was not 100 percent and this was confirmed by the difference between the theoretical and practical percentage of nitrogen. Other absorption bands that were detected can be classified according to table (4).

\section{Elemental Analysis}

Elemental analysis showed a good agreement between theoretical percentage $(12.5 \%)$ and the result of experimental elemental analysis (12\%) as a slight change of about $4.5 \%$.

Table (4) IR Characteristic Absorption bands

\begin{tabular}{|c|c|}
\hline Group & Wave number $\left(\mathbf{c m}^{-1}\right)$ \\
\hline$-\mathrm{OH}$ & 3400 \\
\hline $\mathrm{C}-\mathrm{O}-\mathrm{H}$ & 1435 \\
\hline$-\mathrm{CH}-\mathrm{CH}$ & 2925 \\
\hline $\mathrm{C}-\mathrm{H}$ & 2875 \\
\hline $\mathrm{C}-\mathrm{Cl}$ & 740 \\
\hline $\mathrm{C}-\mathrm{O}-\mathrm{C}$ & 1125 \\
\hline $\mathrm{CH}_{2}-\mathrm{N}_{3}$ & 1281 (bending vibration) \\
\hline $\mathrm{CH}_{2}-\mathrm{O}-\mathrm{CH}_{2}$ & 1665 \\
\hline $\mathrm{CH}_{2}-\mathrm{N}_{3}$ & 2100 (stretching vibration) \\
\hline
\end{tabular}

\section{Water Content}

The optimum water content to be promising in composite solid rocket propellant formulations is about $0.05 \%$. For the prepared AHTPB samples the water content was found to be $0.08 \%$ ,this value was very close to applied in the field of composite solid rocket propellant[15].

\section{Conclusions}

Results of thermochemical calculations were essential for choosing propellant formulations that are candidate for a given practical application to minimize many costly experiments which are used to evaluate the performance and properties of new propellant formulations.

AHTPB was successfully prepared by two steps method with about $15 \%$ conversion. AHTPB prepolymer was characterized through a series of physical and chemical characterizations. Measurement of $0.8516 \mathrm{mg}$. equivalent $\mathrm{OH} / \mathrm{g}$ AHTPB which is very close to that of HTPB (0.8541), indicating that the preparation reactions to produce AHTPB did not affect the terminal hydroxyl groups. Density was determined as $0.967 \mathrm{~g} / \mathrm{cm}^{3}$ which is about $10 \%$ higher than that of HTPB. Viscosity was measured as $5039 \mathrm{cP}$ which is nearly within range of HTPB prepolymer $5306 \mathrm{cP}$ and it was candidate to be used in the field CSRP. AHTPB prepolymer was instrumentally characterized by using infrared spectroscopy to indicate that the azide 
group was inserted in the structure of HTPB and its peak appears at $2100 \mathrm{~cm}^{-1}$. The experimental nitrogen content was found to be $12 \%$ which coincide with the theoretical calculated value, hence complete azidation of the repeating unit of HTPB was occurred. Water content was measured as $0.08 \%$ which is about $15 \%$ higher than that of HTPB and could be accepted from technological point of view.

The characteristics of the examined AHTPB samples gives the prepolymer the opportunity to perform good propellant processing which could be candidate to be used as a binder in solid propellant formulations

\section{References}

[1] Arther Provates, "Energetic Polymers And Plasticizers For Explosive Formulations Areview of Recent Advances", Weapon Systems Division, Aerooutical and Maritine Research Laboratory ,DSTO 2002.

[2] Davens,A, “In Solid Rocket Propulsion Technology ", ,Pergamon Press ,New York ,1993.

[3] Kamal E.M. "The Synthesis of Some Energetic Materials for Solid Rocket Propellants" M.Sc. thesis, MTC, Cairo, 2006.

[4] Borham M.A., "Characterization of Energetic Binders for Explosives" M.Sc. thesis, MTC, Cairo, 1999.

[5] N.Desbiens and V.Dubois, " New developments of the CARTE thermochemical code: I-parameter optimization", EPJ web of conferences, Arpajon, France, 2010.

[6] J.P.Lu, "Evaluation of the thermochemical code-CHEETAH 2.0 for modeling explosives performance", DSTO aeronautical and maritime research laboratory, Australia, 2001.

[7] M.A Sadek, M.A.Radwan, M.A.Borham, "Preparation and Investigation of Azido Hydroxyl Terminated Polybutadiene" $8^{\text {th }}$ international conference on Aerospace Science and Aviation Technology, MTC, Cairo, 1999.

[8] Zakaria T. "Synthesis and Characterization of Energetic Materials for Composite Rocket Propellants" M.Sc. thesis, MTC, Cairo, 2003.

[9] G.Ampleman and F.Beaqre, "Synthesis of Linear GAP Based Thermoplastic Elastomers For Use in Helova Gun Propellant Formulations", (Drev), Coucelette,Quebec, Canada ,1996.

[10] Dr. Mohmed A.Sadek, Dr.M. Shokry Fayed and Hosam A. Mostafa, "Preparation And Characterization of Some Energetic Materials Used in Rocket Propellants and High Explosives",MTC ,Cairo,2002.

[11] I. M. Kamal, M.A.Radwan, H.E. Mostafa and M. A. Borham, "Preperation and Characterization of Glycidyle Azide Polymer (GAP) as a Plasticizer in Composite Solid Rocket Propellant", 2nd ICEE Conference, MTC, Cairo, 2003 November, 2004.

[12] Tony Manzara and Rob Hunter, "Glycidyle Azide Polymer Plasticizer -DSC and Rheological Results", Performance Materials st. Poul, USA, 2000.

[13] Mohmed A.Sadek, Mohamed M. Ismail and Hosam A. Mostafa, "Preparation And Characterization of Some Energetic Materials Used in Rocket Propellants and High Explosives" MTC, Cairo, 1999.

[14] V.Vasudevan and G.Sndararajan "Synthesis of GAP -PB- GAP Triblok Copolymer and Application as Modifier in AP/HTPB Composite Propellant", Departement OF Chemistry, Indian Institute of Technology, Madras India, 2001.

[15] Tsao-Fa Yeh "Thermal Characterization of Propellants Containing GAP Binder", Departement of Applied Chemistry Chung Cheng Institute Of Technology, Tachi, Taiwan 1990. 


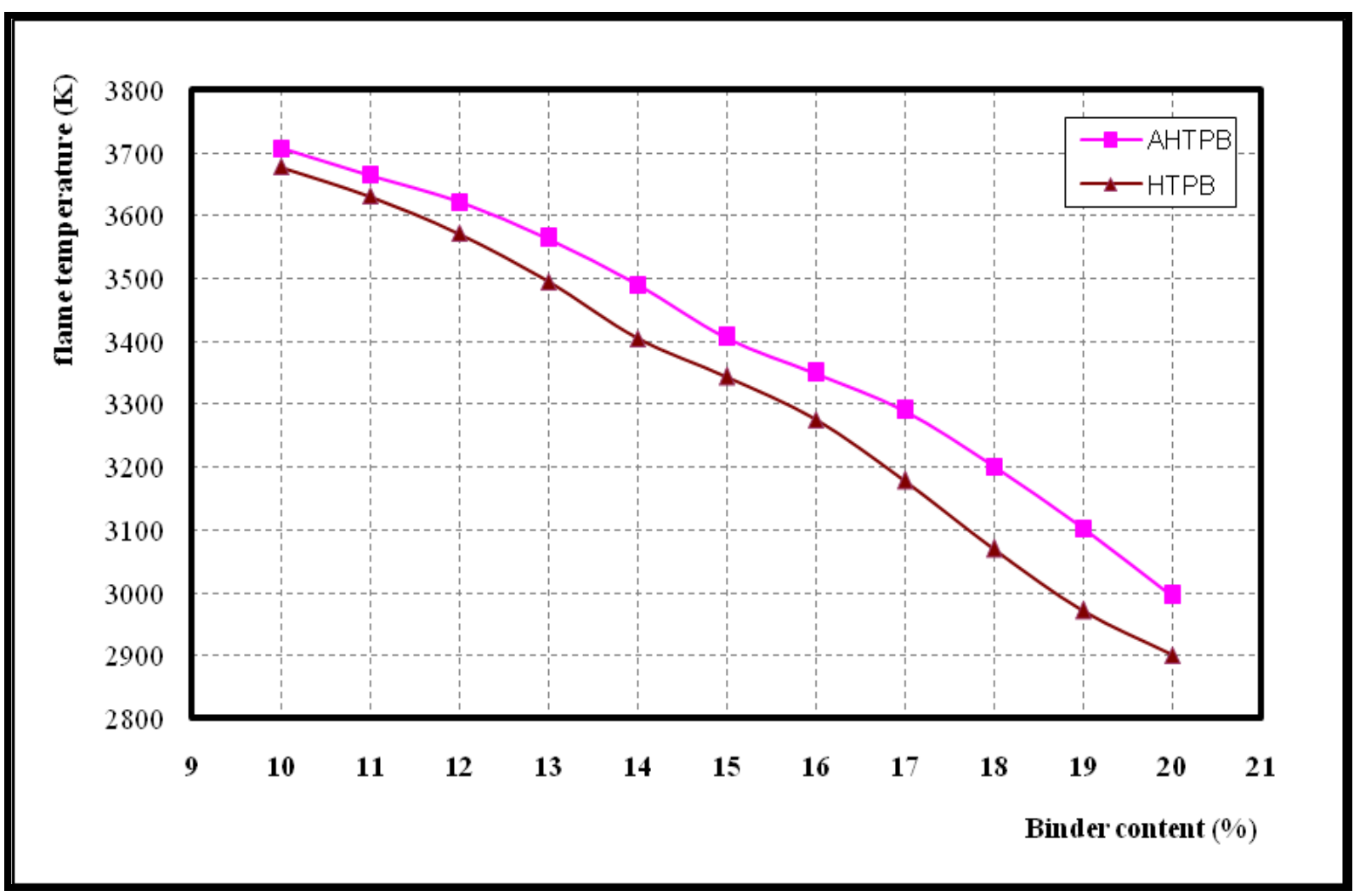

Fig (1) Binder content against flame temperature

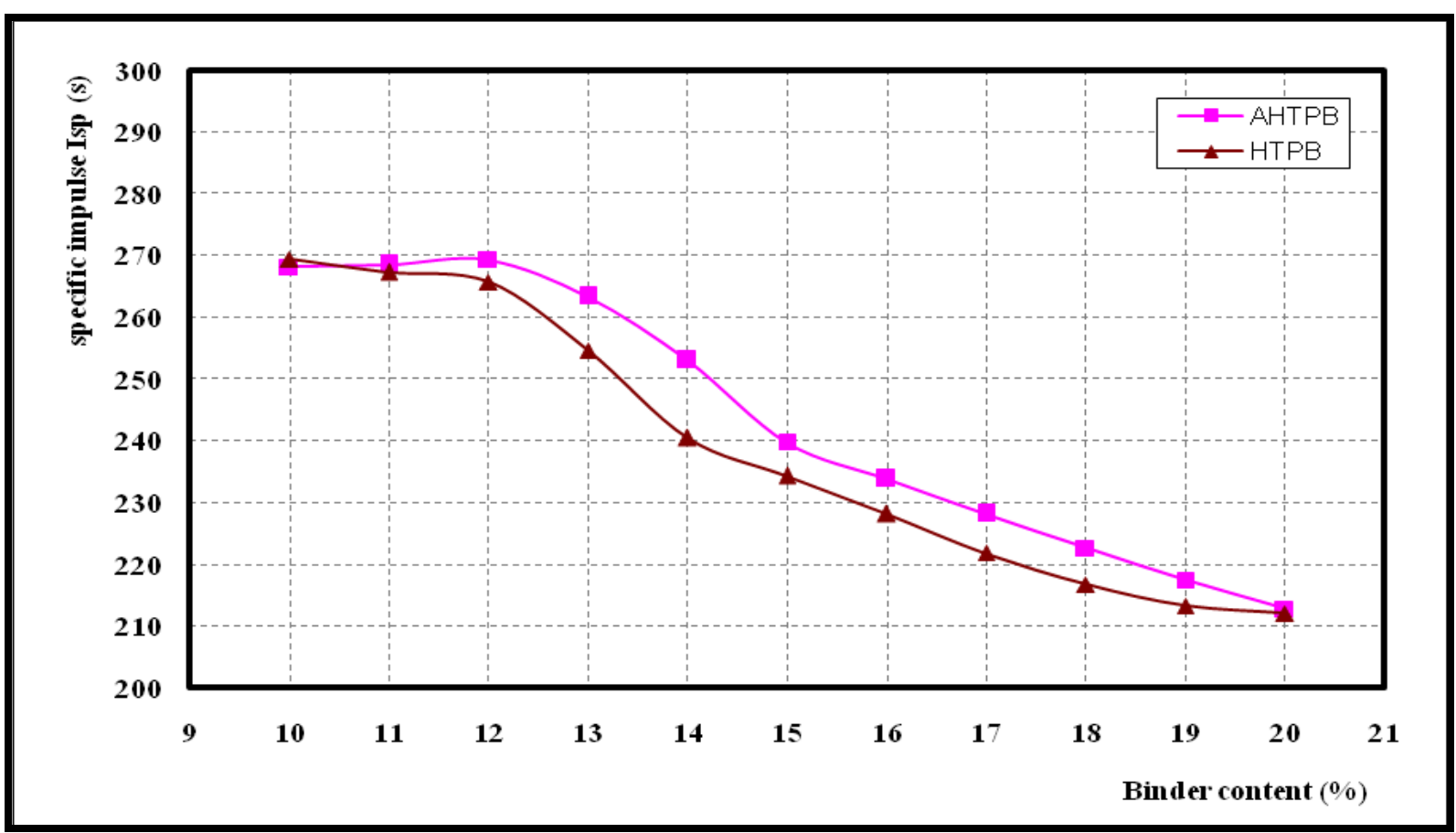

Fig (2) Binder content against specific impulse 


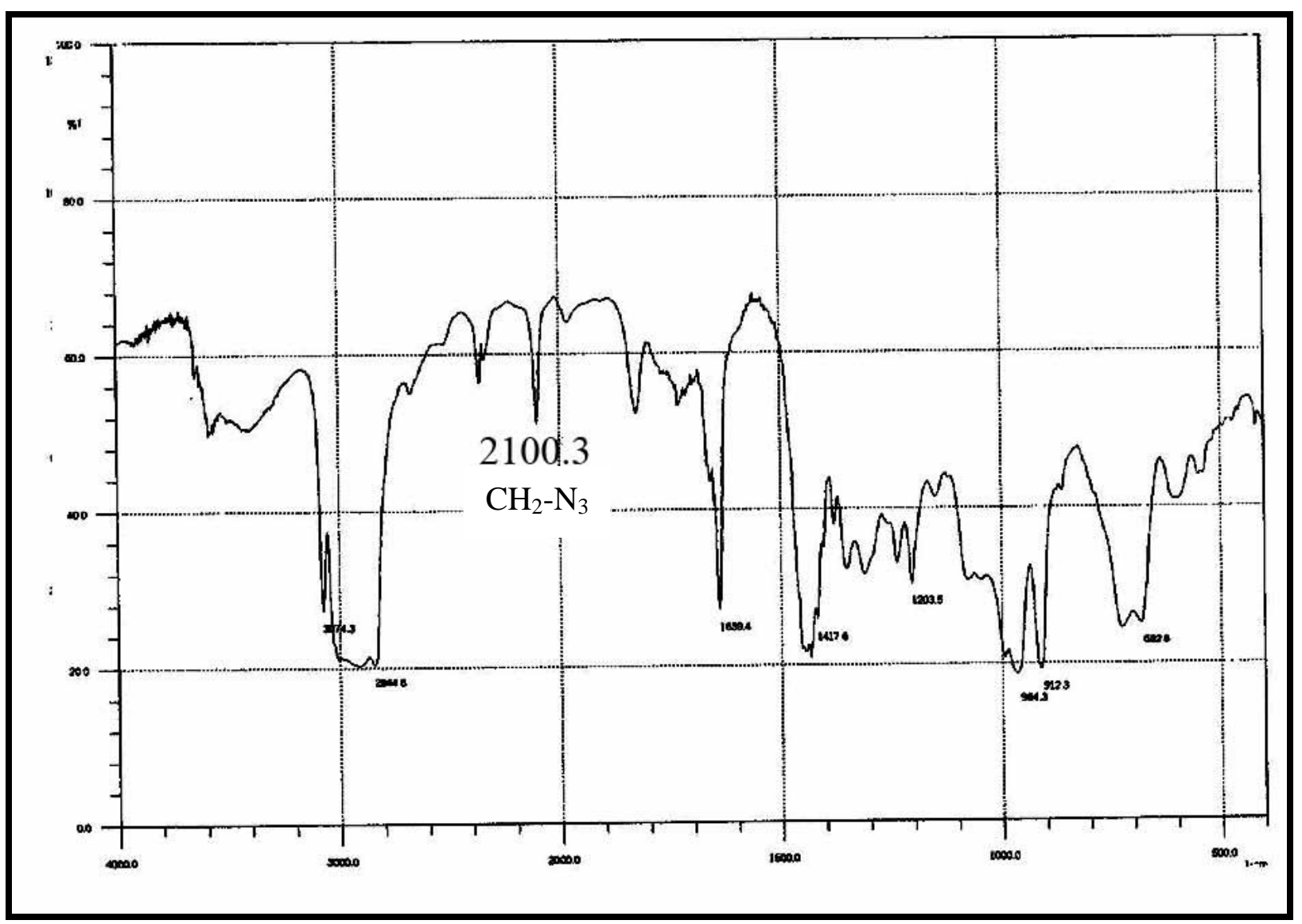

Fig (3) IR Spectrum of AHTPB 